Ann. Zootech., I967, 16 (I), I I-I5.

\title{
EFFETS COMPARÉS D'UN TAMPON-CITRATE ASSOCIÉ AU JAUNE D'CEUF ENTIER OU CHIMIQUEMENT FRACTIONNÉ SUR LA SURVIE IN VITRO DES SPERMATOZOÏDES DE BOUC
}

\author{
J.-Y. CORTEEL \\ avec la collaboration terhnique de F. BartTEall \\ Laboraloire de Physiologie de la Reproduction, \\ Centre de Recherches vétérinaires et zootechniques, 37 -Nouzilly, \\ Cenire expérimental de Sélection et d'Insémination porcine, \\ La Pétinière, 86 - Rouillé
}

\section{SOMMAIRE}

Les effets de trois associations différentes de citrate trisodique et de jaune d'œuf sur la survie in vitro des spermatozoïdes de bouc ont été comparés. Seul parmi les milieux de dilution utilisés, le citrate trisodique associé au précipité acétonique de jaune d'œuf et aux antibiotiques a permis une conservation efficace de la motilité des spermatozoïdes de bouc. I 93 chèvres ont été inséminées avec du sperme dilué dans ce milieu; les résultats obtenus n'ont pas été satisfaisants.

\section{INTRODUC'TION}

A la suite des travaux de Philitips (I939), le vitellus de l'œuf de poule a été très largement utilisé dans les milieux de conservation in vitro des spermatozoïdes de Taureau. Par contre, son utilisation pour le bouc a conduit à des résultats très variables (Buhman, I952; KNOBLAUCH, I952; Bi.okhuis, I959; Singh, I96I ; DAUZIER, I962 et ViAchos, I963). 
Les échecs semblent liés à la dégradation de la molécule de lécithine du jaune d'œuf par un enzyme présent dans le plasma séminal de bouc (Roy, I957); cet enzyme qui scinde la molécule de lécithine du jaune d'œuf en lysolécithine et en acides gras, provoque la coagulation du milieu de conservation (IRITANr et al., I96 à Ig65).

L'analyse de ces phénomènes a permis aux auteurs japonais de montrer que, soumise à l'activité enzymatique du plasma séminal, la fraction du jaune d'œuf insoluble dans l'acétone libérait un taux d'acides gras trois fois moindre que le jaune d'œuf entier non traité. Il nous a paru intéressant d'étudier l'influence respective de différentes fractions du jaune d'oeuf sur le survie in vitro des spermatozoïles de bouc.

\section{MATÉRIEL, E'I MÉTHODES}

Quatre essais de conservation ont lieu successivement à une semaine d"intervalle au cours des mois de janvier et février. Nous disposons de quatre boucs, âgés de 2 à 6 ans soumis à un rythme de collecte bi-hebdomadaire. Le matin de chaque essai, chacun des quatre boucs est collecté deux fois. L'ensemble des collectes a lieu dans un laps de temps aussi bref que possible (20 minutes environ).

La motilité d'ensemble du sperme de chaque éjaculat est appréciée. Lorsqu'ils obtiennent une note au moins égale ou supérieure à 3,5 (échelle de o à 5), les éjaculats sont mélangés pour constituer le volume de sperme nécessaire à chaque essai. 24 éjaculats sont ainsi sélectionnés et utilisés au cours de notre expérience ( 5 pour le premier essai, 6 pour le second, 7 pour le $3^{\mathrm{e}}$ et 6 pour le dernier).

Lors de chaque essai, le pourcentage de spermatozoïdes non colorés est déterminé pour le sperme résultant du mélange des éjaculats retenus. Fractionné en trois volumes égaux, le sperme est alors dilué à $+35^{\circ} \mathrm{C}$ dans trois milieux différents à raison d'un volume de semence pour ro volumes de dilueur. Trois milieux de conservation sont utilisés :

Dilueur $A$ : (itrate trisodique $\left(2 \mathrm{II}_{2} \mathrm{O}\right)$ à 2,9 p. 100 (48 ml); jaune d'œuf frais ( $\mathrm{I} 2 \mathrm{ml}$ ) ; Streptomycine (30 mg); Pénicilline (I $8 \mathrm{mg})$; $\mathrm{pH}$ ajusté à 6,80 .

Dilueur $B$ : Citrate trisodique $\left(2 \mathrm{H}_{2} \mathrm{O}\right)$ à 2,9 p. $100(56,5 \mathrm{ml})$ précipité acétonique de jaune d'œuf $(3,4 \mathrm{~g})$; Streptomycine (30 mg); P'énicilline ( $18 \mathrm{mg}) \mathrm{pH}$ ajusté à 6,80 .

Dilueur C : Citrate trisodicue $\left(2 \mathrm{H}_{2} \mathrm{O}\right)$ à 2,9 p. $100(58,5 \mathrm{ml})$; Phospholipides du jaune d'cuf $(\mathrm{r}, 334 \mathrm{~g})$;

Streptomycine ( $30 \mathrm{mg}$ ); Pénicilline ( $18 \mathrm{mg}) ; \mathrm{pH}$ ajusté à 6,80 .

Le sperme dilué et refroidi (linéairement à raison de $\mathrm{I} 5^{\circ} \mathrm{C}$ heure) est réparti dans des ampoules de verre qui après avoir été scellées à la flamme sont conservées à la chambre froide $\left(+5^{\circ} \mathrm{C}\right)$. Une ampoule par traitement est prélevée le jour de la collecte pour notation de la motilité et détermination du pourcentage de spermatozoïdes non colorés (valeurs portées en Joo sur le graphique). Les examens se poursuivent ensuite tous les deux jours pendant 18 jours.

\section{RÉSULTA'TS}

Au cours des contrôles, le coagulum a été observé dans la dilution $A$ après huit jours de conservation au cours du premier essai et après deux jours dans les trois autres essais. Dans tous les cas l'apparition du coagulum a été suivie de la mort plus ou moins immédiate des spermatozoïdes.

Le dilueur $B$ a permis une survie des spermatozoïdes de $\mathrm{I} 2$ à $\mathrm{I} 6$ jours suivant les essais sans coagulation apparente du milieu. 
Enfin, en l'absence de coagulation, on constate dans le dilueur C une dissociation très nette entre 1'évolution dans le temps du pourcentage de spermatozoïdes non colorés et celle de la motilité : 6 heures après la dilution on n'observe plus que quelques spermatozoïdes animés de mouvements pendulaires, de faible amplitude, alors que le pourcentage de spermatozoïdes non colorés est d'environ $60 \mathrm{p}$. Ioo.

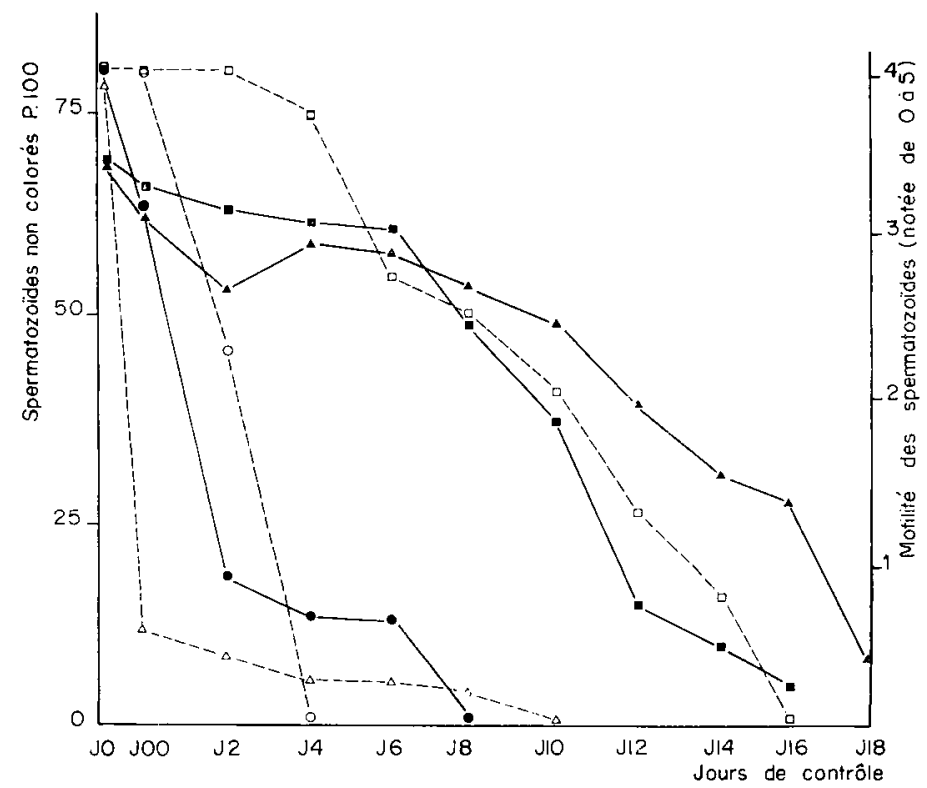

Sumie in vitro des spermatozoildes de Bouc dans différenls milieux de conseration.

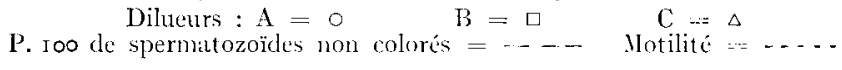

\section{DISCUSSION}

$\mathrm{Au}$ cours de conservations in vitro dans les milieux à base de jaune d'veuf

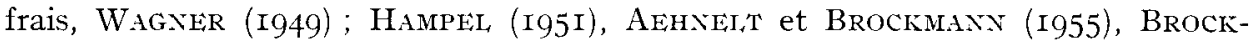
MANN (I956) et NiCOLAI (I957) ont observé des survies de 200 heures.

Au cours de nos essais, la formation du coagulum dans le dilueur A s'est produite après des temps de conservation variables.

Selon Roy (I957), le retard ou même l'absence de réaction enzymatique serait due à un défaut de Calcium, activateur de la réaction. Le plasma séminal et le jaune d'ouf contenant tous deux du Calcium, l'explication de Roy semble devoir être nuancée : des effets de seuils pourraient intervenir ici.

Le dilueur $B$ a permis le maintien cl'une motilité nettement supérieure à celle des témoins.

Dans le milieu contenant les phospholipides du jaune d'œuf, on assiste à une immobilisation immédiate des spermatozoïdes. AAMDAL, LyNGSET et Fossum (1965) obtiennent le même type de réaction en ajoutant I à 2 p. xoo de coagulum ou de la lysolécithine au taux de I p. I ooo au sperme de bouc et de verrat. 
Le maintien d'un haut pourcentage de spermatozoïdes non colorés au cours de la conservation dans le milieu $\mathrm{C}$ ouvre la voie à de nombreuses hypothèses : ou les spermatozoïdes sont morts et leur non coloration serait due à des modifications de la membrane cellulaire inhibant la coloration ou, en l'absence de nécrospermie, s'agit-il plus simplement d'une inhibition réversible ou non de la motilité des spermatozoïdes?

\section{CONCI,USION}

Seul parmi les milieux de dilution utilisés, le tampon citrate associé au précipité acétonique de jaune d'œuf et aux antibiotiques a permis une conservation efficace de la motilité des spermatozoïdes de bouc. Il restait à confirmer la valeur de ce dilueur par des essais de fécondance pratiqués à l'aide de sperme ainsi conservé.

Pour les essais in vivo, le principe des éjactlats partagés n'a pu être maintenu en raison de considérations zootechniques et pratiques.

TABLEAU I

Essai de fécondance

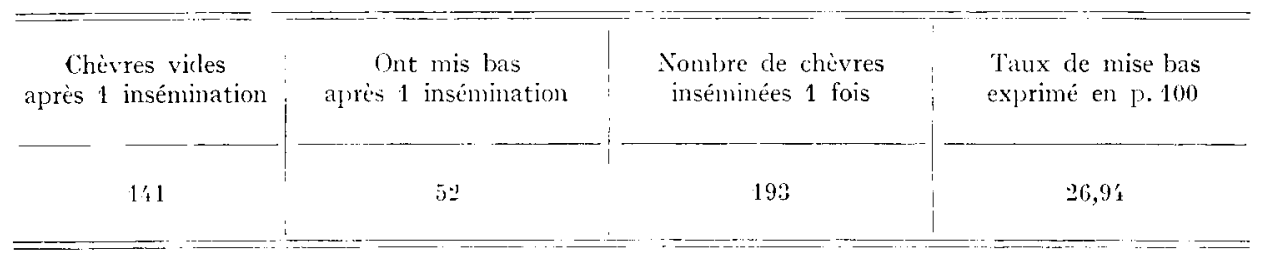

Néanmoins 193 chèvres ont été inséminées par dépôt intravaginal cle $0,4 \mathrm{m1}$ de sperme dilué contenant $1_{5} \mathrm{O} \times I^{6}$ spermatozoïdes. Les résultats, exprimés en nombre de chèvres ayant mis bas après une seule insémination, figurent au tableau I. Ceux-ci sont en moyenne inférieurs de 25 p. Ioo aux résultats obtenus pendant la même saison en utilisant un dilueur à base de lait de vache, écrémé, reconstitué (DAUZIER, I962). Les temps de conservation étaient identiques ( $\leqslant$ I2 h).

Les interactions du plasma séminal de bouc avec les différents constituants du jaune d'ouf sont encore mal connues et méritent d'être précisées. Par ailleurs les essais de fécondance confirment la faible relation entre la survie in vitro et la "fécondance " du sperme de bouc.

Reşu pour publication en janiier 1967.

\section{SUMMARY}

SLRVIVAL OF HE-GOAT SPERMATOZOA IN WHOLE OR FRACTIONATED EGG-YOLK ASSOCIATED WITI A SODIUM CITRATE BUFFER SOLUTION AND ANTIBIOTICS

Whole egg yolk, yolk crude phospholipids and yolk phospholipids associated to yolk proteins have been added to a sodium citrate buffer solution and to antibiotics in an attempt to extend the volume of ejaculated goat semen and to preserve the motility of the spermatozoa at $+4-5^{\circ} \mathrm{C}$. Sperm survival has been checked every other day from collection time through extinction of spermatozoal life. 
The sodium citrate dilutor containing yolk proteins associated to yolk crucle phospholipids proved to be the best for the preservation of sperm motility. Ilowewer when used for the insenination of 193 goats, the results have been unsatisfactory.

\section{RÉFÉRENCES BIBLIOGRAPHIQUES}

Aandal J., Lyngsie O., Fossun K., ig65. 'Toxic effect of lysolecithin on sperm. A preliminary report. Ford Vel. Med., 17, 6.33-634.

Aennelt İ., Brockmanv l'., I955. Zur spermaverdünnung unter Verwendung von eidottern verschiedener hühnerrassen. Dtsch. Tier Wosch., 5, 69- $\imath^{2}$.

Blokiuss J., 1959. Kunstmatige inseminatie bij geiten in de provincie Utrecht. Tijdschr. Diergeneesk., 6, $3+7-35 \mathrm{I}$.

Brocknavn P., 1956. Der cinfluss von eiclotter verschiedener Hühnerrassen auf die lebensdauer von ziegenbock- und bullensperma sowie befruchtungsfähigheit von ziegenbocksperma. Inaug. Diss. Hannov're.

Bumatix K., 1952. Samenübertragung bei ziegen : Verdïnumgs- und Fütterungsversuche. Inaug. Diss. Hannove.

Contres J. M., ig66. Reproduction et lnsémination artilicielle de l'spèce Caprine. Bull. tech. Inform., $210,463-47$ г

DAL71ER L., โ962. Quelques résultats sur l'insémination artificielle caprine. Intern. Incyclopoedia Vet. Stull. r. School vet. Stud. Exlinburgh.

HAMPEL J., I95. Versuche zur verdûnung des ziegenspermas. Inatug. Diss. IIannoare.

Iritani A., Nisinkawa Y., Fekuhara R., ro6r. Studies on the esg-yolk coagulating factor in roat semen. I. Socalisation of the C. F. and decline of pll following coarulation. I'roc. Silver Jubilee: lab. anin. Husband. Coll. Agric. Kyolo, 89-96.

Iritani A., Nisinkawn Y., rg6r. Sturlies on the egg-yolk coagulating factor in goat semen. II. Properties of the coagulating factor and influential conditions for coasulation. Proc. Silaer Jubilee : lab. Anim. Husb. Coll. Igric. Kyolo, 97-104.

Iritail A., NisinkaWa Y., 1963. Studies on the exrryolk coagrulating factor in groat semen. IlI. Release of some acids accompanied by the coagulation phenomena. IV. On the position of yolk constituents attacked by the coagulating enzyme. V. Purification of the egg-yolk coagulating enzyme. Jap. J. anim. Reprod., 8, 100-121.

IRItani A., Nismlkawa Y., tg64. Studies on the egg-yolk coagulating enzyme in goat semen. VI. On the chemical properties of the ejaculated semen and the secretion of accessory sexual organs in the croat VII. Variations in the enzyne activity of the semen between breeding and non breeding season and in each ejaculate. VIII. The enzyme activity of the semen collected by electrical stimulation and the semen depleted by the artificial vagina. Jap. J. amim. Reprod., 10, $44^{-62}$.

Knoblauci H., 1952. Untersuchungen an ziegrenbockejaculaten. Zuchihyg., 6, 9-22.

Xicolat E., 1957. Verdünmmgs und besimumgsversuche (Riedel de Haen-Verdumer D) Sowie Fütterungs versuche. Inaug. Diss. Hannovre.

Phillips P. H., 1939. The preservation of Bull semen. J. biol. Chem., 130, 4I5.

Roy A., 1957. Fogr yolk coagulating enzyme in the semen and cowper's gland of the goat. Nalure, 179, $3 \mathrm{I} 8$.

Ṡxon G., г96. Observations sur l'application pratique de linsémination artiticielle chez les bovins, les buffles, les chèvres à Izatnaşrar (Inde). Ann. Zoolech., 10, 229-232.

VLAchos K., I963. Cintersuchungen über die erfolge kunstlicher besamung der ziegen in nordgriechenland während cles Jahres ig6r. Tuchllygg., 7, $21+-224$.

Wagner 11, ry+9). Erfahrungen und versuche in ter ziegren-besamung. Inaug. Diss. Hanove. 